\title{
Association of food consumption with total volumes of visceral and subcutaneous abdominal adipose tissue in a Northern German population
}

\author{
Daniela Rüttgers ${ }^{1 *}$, Karina Fischer ${ }^{1}$, Manja Koch ${ }^{2}$, Wolfgang Lieb ${ }^{2,3}$, Hans-Peter Müller ${ }^{4}$, Gunnar Jacobs ${ }^{4}$, \\ Jan Kassubek ${ }^{3}$ and Ute Nöthlings ${ }^{1}$ \\ ${ }^{1}$ Department of Nutrition and Food Sciences, University of Bonn, 53115 Bonn, Germany \\ ${ }^{2}$ Institute of Epidemiology, Christian-Albrechts University Kiel, 24105 Kiel, Germany \\ ${ }^{3}$ PopGen Biobank, University Hospital Schleswig-Holstein, 24105 Kiel, Germany \\ ${ }^{4}$ Department of Neurology, University of Ulm, 89081 Ulm, Germany
}

(Submitted 14 January 2015 - Final revision received 21 July 2015 - Accepted 25 August 2015 - First published online 7 October 2015)

\section{Abstract}

Excess accumulation of visceral adipose tissue (VAT) is a known risk factor for cardiometabolic diseases; further, subcutaneous abdominal adipose tissue (SAAT) and the ratio of both (VAT:SAAT ratio) have been discussed as potentially detrimental. Information about the association between diet and adipose tissue is scarce. This study aimed to identify food group intake associated with VAT and SAAT and the VAT:SAAT ratio in a Northern German population. A cross-sectional analysis was conducted in 344 men and 241 women who underwent an MRI to quantify total volumes of VAT and SAAT. Intake of fourteen food groups was assessed with a self-administered 112-item FFQ. Linear regression models adjusted for age, sex, energy intake, physical activity, intake of other food groups and mutual adjustment for VAT and SAAT were calculated to analyse the associations between standardised food group intake and VAT and SAAT, or the VAT:SAAT ratio. Intakes of potatoes $(P=0.043)$ and cakes $(P=0.003)$ were positively and inversely, respectively, associated with both VAT and SAAT. By contrast, intake of cereals was negatively associated with VAT $(P=0.045)$ only, whereas intakes of eggs $(P=0.006)$ and non-alcoholic beverages $(P=0.042)$ were positively associated with SAAT only. The association between eggs and non-alcoholic beverages with SAAT remained significant after further consideration of VAT. Intake of non-alcoholic beverages was also inversely associated with the VAT:SAAT ratio $(P=0 \cdot 001)$. Our analysis adds to the evidence that intake of foods is independently associated with VAT or SAAT volumes.

Key words: Visceral adipose tissue: Subcutaneous adipose tissue: Food group consumption: MRI

Visceral adiposity, characterised by excess accumulation of visceral adipose tissue (VAT) adipose tissue (AT) has been recognised as a risk factor for metabolic diseases, including fatty liver disease, type 2 diabetes and $\mathrm{CVD}^{(1)}$. Visceral adiposity and its consequences have become a growing public health problem worldwide mainly due to increasing sedentary lifestyles and detrimental changes in dietary behaviour ${ }^{(1)}$. The association of subcutaneous AT with CVD and metabolic diseases is less clear ${ }^{(2,3)}$ and may depend on its distribution on the human body. Although a protective influence of thigh subcutaneous AT on ectopic energy storage and markers of chronic inflammation has been suggested ${ }^{(4,5)}$, the role of subcutaneous abdominal adipose tissue (SAAT) in cardiometabolic risk is not conclusive ${ }^{(6,7)}$. Data from the Framingham Heart Study suggest that not only the absolute quantity of VAT, but also the proportion of VAT to SAAT, the VAT:SAAT ratio, which reflects the disposition to store energy in ectopic fat compartments, may be an independent risk factor for cardiometabolic disease ${ }^{(8)}$.

VAT adipocytes differ from SAAT adipocytes in their physiology and metabolism, such as uptake of glucose and circulating free fatty acids ${ }^{(9)}$. Energy storage in VAT or SAAT adipocytes might therefore be differently influenced by food intake. Individual studies on food intake and AT distribution have reported a positive association of the consumption of eggs, sweetened beverages ${ }^{(10)}$, beer and French-fried potatoes $^{(11)}$ with central obesity measured by waist circumference. Anthropometric surrogates of fat distribution, however, do not allow the differentiation between VAT and SAAT, or any classification of individuals with high amounts of VAT or SAAT ${ }^{(12,13)}$. By contrast, imaging techniques such as computed tomography (CT) or MRI enable accurate distinction between VAT and SAAT. Results from a study of Latino adolescents discriminating between VAT and SAAT have shown

Abbreviations: AT, adipose tissue; EPIC, European Prospective Investigation into Cancer and Nutrition; SAAT, subcutaneous abdominal adipose tissue; VAT, visceral adipose tissue.

* Corresponding author: D. Rüttgers, fax +49228 73 60492, email d.moewes@uni-bonn.de 
that intake of dietary fibre was inversely associated with VAT in overweight Latino youth ${ }^{(14-16)}$. Similarly, whole-grain intake was inversely associated with VAT and SAAT in adults, whereas refined-grain intake was positively associated with VAT in adult participants of the Framingham Heart Study ${ }^{(17)}$. Nonetheless, studies relating food intake or other aspects of diet to MRI- or CT-determined total volumes of VAT and SAAT instead of to anthropometric surrogates or single-slice scans of abdominal AT are limited and often reduced to the association of single nutrients with VAT and SAAT ${ }^{(18)}$. The aim of the present study was therefore to investigate whether intakes of food groups were associated with MRI determined total volumes of VAT and SAAT, as well as with the VAT:SAAT ratio. Furthermore, we examined whether the observed associations were the same for both AT compartments or altered by mutual adjustment for VAT and SAAT, respectively. Next to metabolic differences, VAT and SAAT adipocytes differ in the amount of synthesised and secreted adipokines. The release of adipokines such as leptin, adiponectin and C-reactive protein (CRP) is altered in obesity ${ }^{(9)}$ and might influence storage of energy preferred in VAT or SAAT. Therefore, we investigated whether the associations between food groups and AT were modified by BMI.

\section{Methods}

\section{Study design and population}

The study was a cross-sectional analysis using data from the first follow-up examination of the PopGen control cohort, which is a population-based study of 1316 adults recruited into the PopGen Biobank in Kiel, Northern Germany ${ }^{(19)}$. Baseline examinations were performed between October 2005 and May 2006 on 747 participants based on a population registry sampling in Kiel. In addition, 569 blood donors were recruited via the Schleswig-Holstein University Medical Center. The first follow-up of the PopGen control cohort was conducted between 2010 and 2012. Study participants were invited by mail, and 930 individuals agreed to participate in the follow-up visit. As part of the phenotypic assessment, 653 participants (59\% males) underwent whole-body MRI scanning. All participants provided a written informed consent to the study. The study was approved by the ethics committee of the University of Kiel, Germany ${ }^{(20)}$. Of the 653 participants who underwent covariate and outcome assessment, ten participants were excluded because of implausible energy intakes and fifty-seven participants because of major MRI artefacts. Defined limits for exclusion were energy intakes $<2.5 \mathrm{MJ} / \mathrm{d}$ for both sexes or $>17.5 \mathrm{MJ} / \mathrm{d}$ in men and $>16.74 \mathrm{MJ} / \mathrm{d}(4000 \mathrm{kcal} / \mathrm{d})$ in women $^{(17)}$. Data of the remaining 585 participants (241 women and 344 men) were included in all statistical analyses.

\section{Dietary assessment}

Usual dietary intakes over the past year were assessed using a self-administered semi-quantitative FFQ specifically designed for the German population ${ }^{(21)}$. Energy intakes were estimated using the German Food Code and Nutrient Data Base (version II.3) ${ }^{(22)}$. The FFQ was web-based but optionally also available as a paper version. The primary FFQ was developed and validated as part of the dietary assessment in the European Prospective Investigation into Cancer and Nutrition (EPIC)Potsdam study ${ }^{(23)}$. The FFQ used in the PopGen control cohort was shortened from 146 to 112 food items, which mainly explained the variation in nutrient and food intake to ensure high response rates in dietary assessment ${ }^{(21,24)}$. Food items were aggregated into fourteen food groups: (1) potatoes; (2) vegetables, including leafy, fruit and root vegetables, cabbages, onions and mushrooms; (3) legumes; (4) fruits, including fruits, nuts and seeds; (5) dairy products, including milk and milk drinks, yoghurt and pudding, curd, cheese and coffee cream; (6) cereals and cereal products, including flour, flakes, pasta, rice, bread, breakfast cereal and salty biscuits; (7) meat and meat products, including beef, pork, poultry, sausages, cold cuts and offals; additionally to overall meat intake, meat intake was further subdivided into intake of red and processed meat and intake of white meat; (8) fish and fish products; (9) eggs; (10) fat, including vegetable oils, butter, margarine and chip fat; (11) sugar and confectionery, including sugar, honey, jam, chocolate and sweets, syrup and ice cream; (12) cake, including cake, pastries and cookies; (13) nonalcoholic beverages, including fruit juice and lemonades, coffee, tea, herbal tea and water; additionally to overall non-alcoholic beverage intake, the food group was further subdivided into sugar-sweetened beverages (lemonades and juices), tea and coffee; (14) alcoholic beverages, including wine and dessert wine, beer, spirituous beverages, aniseed beverages, liqueur and cocktails. The food groups sauces, soups and miscellaneous were not considered for the current analysis because of the high variability in the composition of the individual group items.

\section{Covariate assessment}

Baseline and follow-up assessments were conducted at the PopGen study centre in Kiel, Germany. Participants underwent physical examinations and blood sampling as well as filled in self-administered questionnaires on medical history. Anthropometric assessments were conducted by trained study nurses. Weight was measured in light clothing without shoes. To correct for clothing, $2 \mathrm{~kg}$ were subtracted. Height was measured without shoes with the participant standing in an upright position. BMI was calculated as weight in kilograms divided by height in metres squared. Further data on sex, age and lifestyle factors such as smoking habits, alcohol consumption and physical activity were collected using self-administered questionnaires ${ }^{(25)}$. Physical activity was evaluated as described previously ${ }^{(25)}$.

\section{Outcome assessments}

Participants underwent MRI covering the whole body from wrist to ankle on a 1.5-T scanner (Magnetom Avanto; Siemens Medical Systems) in a supine position with hands above their head. To minimise the occurrence of breathing motion artefacts, participants were required to hold their breath during the t1-weighted gradient-echo sequence (repetition time $157 \mathrm{~ms}$, time to echo $4 \mathrm{~ms}$, flip angle $70^{\circ}$ ). Transversal two-dimensional 
MRI slices with a thickness of 8 and $2 \mathrm{~mm}$ interslice gaps were obtained. Pre-processing and analysis of both VAT and SAAT were conducted by the semi-automatic software package Automatic Tissue Labelling Analysis Software (ATLAS), which allows to merge all slices to a three-dimensional continuous data set ${ }^{(26)}$. Intensity homogenisation was performed by an interactive repair function. For segmentation of both VAT and SAAT, the same intensity-based threshold algorithm was selected. Application of the Adapted Rendering for Tissue Intensity Segmentation algorithm allowed to automatically distinguish between VAT and SAAT voxels; the segmentation process has been described in detail elsewhere ${ }^{(26,27)}$. SAAT was determined as the sum of subcutaneous AT voxels underneath the skin layers surrounding the abdomen from top of the liver to the femur heads. VAT was determined as the sum of VAT voxels from the top of the liver to the femur heads and the abdominal muscular wall as anatomical border. During post-processing, liver fat and fat in the intestinal loops were manually excluded from VAT segmentation, and minor MRI artefacts, which were mainly caused by hip implants and stents, were corrected. In the case of major non-corrigible MRI artefacts, with breathing motion being the main cause, participants were excluded from further analysis. The voxel size $(3.9 \times 2 \times 8 \mathrm{~mm})$ was multiplied with the number of voxels to get the volumes (in $\mathrm{dm}^{3}$ ) of VAT and SAAT. The ratio of VAT:SAAT was calculated by dividing the volume of VAT by the SAAT volume. To minimise the effect of interrater-variability, all ATLAS-based analyses were conducted by the same observer. In a subset of thirty-eight participants, the accuracy of semi-automatically determined volumes of VAT and SAAT was validated against manually determined volumes using the image analysis software sliceO-matic version 4.2 (Tomovision). AT volumes analysed by ATLAS and slice-O-matic showed a high intraclass correlation for VAT $(r$ 0.996) and SAAT ( $r$ 0.996), respectively.

\section{Statistical analysis}

Descriptive statistics for characterisation of the study population are presented as means and standard deviations and dietary intakes as medians and interquartile ranges. Normality of distribution was tested by the Shapiro-Wilk test. AT variables were used as continuous variables and natural logarithmically (SAAT) or square root transformed (VAT) to approximate normal distribution. Pearson's correlation coefficients were calculated for AT variables and BMI. Multiple linear regression models were used to assess the association between food group intake and VAT and SAAT, or the VAT:SAAT ratio, with food groups as the exposure and the AT measurements as outcome variables. Covariates of the first regression model were age, sex, total energy intake and physical activity. A second model was additionally adjusted for all other food groups, and a third model was additionally mutually adjusted for VAT or SAAT. All models were stratified by median BMI $\left(<26.7\right.$ and $\left.\geq 26.7 \mathrm{~kg} / \mathrm{m}^{2}\right)$. The regression models were not stratified by sex because no effect modification by sex on either AT was observed. The variance inflation factor was used to test for multicollinearity in regression models adjusted for other food groups. Subgroups of meat intake and non-alcoholic beverage intake were not considered in the second or third model, because the variance inflation factor indicated high collinearity in these models. $P$ values $<0.05$ were considered statistically significant, and all $P$ values were reported two-sided. All statistical analyses were conducted with SAS statistical analysis software, version 9.3 (SAS Institute Inc.).

\section{Results}

Characteristics of the study participants are presented by sex-specific quartiles of VAT (Table 1) and SAAT (Table 2). The mean BMI was $26.8(\mathrm{sD} 5.3) \mathrm{kg} / \mathrm{m}^{2}$ in women and $27.4(\mathrm{sD} 3.7) \mathrm{kg} / \mathrm{m}^{2}$ in men. Women had lower volumes of VAT $\left(2.5(\mathrm{SD} 1.7) v .4 .7(\mathrm{SD} 2.5) \mathrm{dm}^{3}\right)$ and higher volumes of SAAT (7.6 (sD 4.7) v. $5.7\left(\mathrm{sD} \mathrm{3.0)} \mathrm{dm}^{3}\right.$ ) compared with men, which resulted in a lower VAT:SAAT ratio of 0.35 (SD 0.16) compared with 0.82 (SD 0.34) in men (data not shown). With increasing amounts of VAT, participants were older, had a higher body weight, waist circumference, systolic and diastolic blood pressure, whole-blood glycated $\mathrm{Hb}$ (HbA1c) and plasma TAG, and lower HDL-cholesterol $\left(P_{\text {trend }}<0.001\right)$. Furthermore, with increasing VAT, subjects had higher intakes of potatoes and lower intakes of fruits, cereals, fat and sugar $\left(P_{\text {trend }}<0 \cdot 05\right)$. With increasing amounts of SAAT, participants had a higher body weight, waist circumference, waist:hip ratio, systolic and diastolic blood pressure, whole-blood HbA1c and plasma TAG, and lower HDL-cholesterol as well as lower reported energy intake $\left(P_{\text {trend }}<0 \cdot 01\right)$. Moreover, with increasing amounts of SAAT, subjects had significantly higher intakes of eggs and lower intakes of fruits, cereals, fat, sugar and cake $\left(P_{\text {trend }}<0 \cdot 01\right)$. In addition, we observed a positive correlation between both SAAT $(r$ 0.84; $P<0.001)$ and VAT $(r$ 0.67; $P<0.001)$ and BMI. However, there was no statistically significant correlation between the VAT:SAAT ratio $(r-0 \cdot 01$; $P=0.77$ ) and BMI (data not shown).

In unstratified analysis adjusted for sex, age, energy intake and physical activity (model 1), VAT was significantly positively associated with intakes of potatoes, total meat, red and processed meat, white meat, eggs and alcoholic beverages, whereas intakes of dairy products, cereals, sugar and confectionary, cakes and tea were negatively associated with VAT (Table 3). After additional adjustment for other food groups (model 2), the positive association between overall meat intake and VAT and the negative association between dairy products and sugar and confectionery, respectively, and VAT was no longer significant. After adjustment for SAAT (model 3), a negative association between non-alcoholic beverages and VAT occurred. Stratified by median BMI, the intakes of dairy products, cereals and cakes were inversely associated with VAT in the BMI $<26.7 \mathrm{~kg} / \mathrm{m}^{2}$ stratum, and the intake of sugar and confectionery and legumes was negatively and positively associated, respectively, with VAT in the $\mathrm{BMI} \geq 26.7 \mathrm{~kg} / \mathrm{m}^{2}$ stratum. Significant effect modification by BMI on VAT was observed for legumes, dairy products and cakes. In unstratified SAAT models adjusted for sex, age, energy and physical activity, intakes of potatoes, total meat, red and processed meat and white meat, eggs, and non-alcoholic beverages were positively associated with SAAT, whereas intakes of sugar and 
Table 1. Characteristics of the study population by sex-specific quartiles (Q) of visceral adipose tissue (VAT) (Mean values and standard deviations; 585 subjects)*

\begin{tabular}{|c|c|c|c|c|c|c|c|c|c|}
\hline & \multicolumn{8}{|c|}{ VAT categories } & \multirow[b]{3}{*}{$P_{\text {trend }}$} \\
\hline & \multicolumn{2}{|c|}{ Q1 } & \multicolumn{2}{|c|}{ Q2 } & \multicolumn{2}{|c|}{ Q3 } & \multicolumn{2}{|c|}{ Q4 } & \\
\hline & Mean & SD & Mean & SD & Mean & SD & Mean & SD & \\
\hline$n$ & \multicolumn{2}{|c|}{146} & \multicolumn{2}{|c|}{146} & \multicolumn{2}{|c|}{147} & \multicolumn{2}{|c|}{146} & \\
\hline VAT $\left(\mathrm{dm}^{3}\right)$ & & & & & & & & & \\
\hline Males & 2.6 & 0.7 & $4 \cdot 1$ & 0.3 & $5 \cdot 3$ & 0.4 & 7.8 & 1.4 & \\
\hline Females & 1.4 & 0.3 & $2 \cdot 1$ & 0.2 & 2.9 & 0.3 & $5 \cdot 0$ & 1.3 & \\
\hline Age (years) & $57 \cdot 0$ & 13.9 & $60 \cdot 0$ & 11.9 & $62 \cdot 1$ & $10 \cdot 9$ & 64.0 & 11.7 & $<0.001$ \\
\hline Weight $(\mathrm{kg})$ & 69.1 & $12 \cdot 2$ & $77 \cdot 3$ & 11.3 & 82.3 & 11.6 & 93.5 & $16 \cdot 7$ & $<0.001$ \\
\hline Height $(\mathrm{cm})$ & 171.9 & 10.5 & $171 \cdot 7$ & 9.8 & $171 \cdot 7$ & 9.4 & $172 \cdot 0$ & $10 \cdot 7$ & 0.937 \\
\hline BMI $\left(\mathrm{kg} / \mathrm{m}^{2}\right)$ & 23.3 & 2.5 & $26 \cdot 2$ & 2.5 & $27 \cdot 9$ & 2.9 & 31.5 & 4.6 & $<0.001$ \\
\hline Waist circumference $(\mathrm{cm})$ & 83.8 & 9.2 & 92.8 & 8.5 & $99 \cdot 2$ & $8 \cdot 3$ & $108 \cdot 7$ & 11.0 & $<0.001$ \\
\hline Waist:hip ratio & 0.9 & 0.1 & 0.9 & 0.1 & 1.0 & 0.1 & 1.0 & 0.1 & $<0.001$ \\
\hline Systolic blood pressure (mmHg) & $130 \cdot 8$ & $15 \cdot 6$ & 131.4 & $16 \cdot 1$ & $135 \cdot 1$ & $16 \cdot 7$ & $140 \cdot 7$ & $17 \cdot 4$ & $<0.001$ \\
\hline Diastolic blood pressure $(\mathrm{mmHg})$ & $78 \cdot 1$ & 9.0 & $80 \cdot 2$ & $8 \cdot 3$ & $82 \cdot 1$ & 8.9 & 84.9 & $10 \cdot 0$ & $<0.001$ \\
\hline Whole-blood HbA1c (\%) & 5.5 & 0.4 & 5.6 & 0.5 & 5.7 & 0.5 & 6.0 & 0.8 & $<0.001$ \\
\hline C-reactive protein $(\mathrm{nmol} / \mathrm{l}) \dagger$ & $56 \cdot 2$ & 131.4 & 23.8 & 29.5 & $26 \cdot 7$ & $22 \cdot 8$ & $47 \cdot 6$ & $66 \cdot 6$ & 0.754 \\
\hline Plasma HDL-cholesterol $(\mathrm{mmol} / \mathrm{l})$ & $2 \cdot 0$ & 0.5 & 1.8 & 0.5 & 1.7 & 0.5 & 1.5 & 0.4 & $<0.001$ \\
\hline Plasma LDL-cholesterol (mmol/l) & 3.3 & 0.9 & 3.4 & 0.8 & 3.6 & 1.0 & 3.4 & 0.9 & 0.050 \\
\hline Plasma TAG (mmol/l) & 1.0 & 0.6 & 1.3 & 0.7 & 1.4 & 0.8 & 1.7 & 1.1 & $<0.001$ \\
\hline Energy intake $(\mathrm{kJ} / \mathrm{d})$ & 9811 & 2656 & 9527 & 2506 & 9195 & 2373 & 9219 & 2581 & 0.022 \\
\hline Physical activity (MET-h/week) & 113.1 & $61 \cdot 1$ & $110 \cdot 0$ & $70 \cdot 8$ & $110 \cdot 8$ & 78.4 & $100 \cdot 5$ & $75 \cdot 6$ & 0.139 \\
\hline Potatoes $(\mathrm{g} / \mathrm{d})$ & $74 \cdot 3$ & 44.5 & 82.4 & 43.4 & $87 \cdot 1$ & 45.9 & 91.8 & $45 \cdot 1$ & 0.001 \\
\hline Vegetables $(\mathrm{g} / \mathrm{d})$ & 201.7 & $100 \cdot 9$ & $196 \cdot 0$ & $88 \cdot 1$ & 191.9 & $77 \cdot 2$ & $206 \cdot 7$ & $90 \cdot 3$ & 0.745 \\
\hline Legumes $(\mathrm{g} / \mathrm{d})$ & $3 \cdot 2$ & 3.3 & 2.7 & 3.0 & 3.4 & 3.7 & 3.3 & 3.2 & 0.354 \\
\hline Fruits $(\mathrm{g} / \mathrm{d})$ & 254.6 & 167.6 & $227 \cdot 2$ & 133.8 & $208 \cdot 2$ & $126 \cdot 8$ & 224.9 & 142.6 & 0.042 \\
\hline Dairy products $(\mathrm{g} / \mathrm{d})$ & $279 \cdot 3$ & $166 \cdot 9$ & $247 \cdot 8$ & $155 \cdot 3$ & 240.4 & 142.5 & $247 \cdot 1$ & $142 \cdot 1$ & 0.065 \\
\hline Cereals $(\mathrm{g} / \mathrm{d})$ & 184.5 & 96.9 & 166.9 & 103.8 & $149 \cdot 9$ & $70 \cdot 4$ & 146.9 & $72 \cdot 1$ & $<0.001$ \\
\hline Meat $(\mathrm{g} / \mathrm{d})$ & $109 \cdot 7$ & $75 \cdot 3$ & $121 \cdot 7$ & 67.7 & 120.3 & 73.5 & 124.5 & $64 \cdot 1$ & 0.100 \\
\hline Fish $(\mathrm{g} / \mathrm{d})$ & $31 \cdot 1$ & 23.1 & 28.8 & 23.6 & 30.0 & $22 \cdot 1$ & 29.7 & 22.5 & 0.722 \\
\hline Eggs $(g / d)$ & $15 \cdot 2$ & 11.6 & 17.4 & 11.9 & $19 \cdot 0$ & $12 \cdot 1$ & 17.5 & 11.9 & 0.054 \\
\hline Fat and oils (g/d) & 38.8 & 21.0 & 38.8 & $22 \cdot 1$ & 33.5 & 14.0 & 34.0 & 14.5 & 0.004 \\
\hline Sugar and confectionery $(\mathrm{g} / \mathrm{d})$ & 53.1 & $27 \cdot 3$ & $51 \cdot 2$ & $24 \cdot 6$ & 50.5 & $26 \cdot 6$ & $47 \cdot 0$ & 24.0 & 0.046 \\
\hline Cake $(g / d)$ & 68.3 & 49.5 & $57 \cdot 1$ & 34.6 & 54.7 & 43.2 & 61.6 & 41.5 & 0.153 \\
\hline Non-alcoholic beverages $(\mathrm{g} / \mathrm{d})$ & $2173 \cdot 1$ & $869 \cdot 8$ & 1953.8 & $935 \cdot 2$ & 2051.6 & $908 \cdot 1$ & 1988.3 & 899.1 & 0.173 \\
\hline Alcoholic beverages $(\mathrm{g} / \mathrm{d})$ & $212 \cdot 3$ & 328.9 & 219.9 & $259 \cdot 2$ & 258.9 & $270 \cdot 1$ & $250 \cdot 1$ & $515 \cdot 2$ & 0.249 \\
\hline
\end{tabular}

$\mathrm{HbA1c}$, glycated $\mathrm{Hb}$; MET, metabolic equivalent task.

* $P<0.05$ was considered statistically significant. $P$ values are reported two-sided. The median value in each quartile category was used as a variable in a linear regression as a test for trend.

† Information available on 358 subjects. 
Table 2. Characteristics of the study population by sex-specific quartiles $(Q)$ of subcutaneous abdominal adipose tissue (SAAT) (Mean values and standard deviations; 585 subjects) ${ }^{\star}$

\begin{tabular}{|c|c|c|c|c|c|c|c|c|c|}
\hline & \multicolumn{8}{|c|}{ SAAT categories } & \multirow[b]{3}{*}{$P_{\text {trend }}$} \\
\hline & \multicolumn{2}{|c|}{ Q1 } & \multicolumn{2}{|c|}{ Q2 } & \multicolumn{2}{|c|}{ Q3 } & \multicolumn{2}{|c|}{ Q4 } & \\
\hline & Mean & SD & Mean & SD & Mean & SD & Mean & SD & \\
\hline$n$ & \multicolumn{2}{|c|}{146} & \multicolumn{2}{|c|}{146} & \multirow{2}{*}{\multicolumn{2}{|c|}{147}} & \multirow{2}{*}{\multicolumn{2}{|c|}{146}} & \\
\hline SAAT $\left(\mathrm{dm}^{3}\right)$ & & & & & & & & & \\
\hline Males & 3.4 & 0.9 & $5 \cdot 1$ & 0.3 & $6 \cdot 4$ & 0.5 & 9.6 & $2 \cdot 6$ & \\
\hline Females & 3.8 & 1.0 & 6.4 & 0.7 & 8.8 & 0.7 & 13.6 & 3.6 & \\
\hline Age (years) & $61 \cdot 8$ & 11.5 & $60 \cdot 3$ & $12 \cdot 1$ & $62 \cdot 1$ & $13 \cdot 0$ & $59 \cdot 0$ & $12 \cdot 7$ & 0.151 \\
\hline Weight (kg) & $67 \cdot 2$ & $10 \cdot 6$ & $75 \cdot 7$ & $10 \cdot 6$ & $82 \cdot 4$ & 9.7 & $96 \cdot 8$ & 14.4 & $<0.001$ \\
\hline Height $(\mathrm{cm})$ & $170 \cdot 4$ & 9.8 & $172 \cdot 0$ & $10 \cdot 0$ & $172 \cdot 0$ & $10 \cdot 1$ & $172 \cdot 8$ & 10.4 & 0.052 \\
\hline $\mathrm{BMI}\left(\mathrm{kg} / \mathrm{m}^{2}\right)$ & 23.1 & 2.4 & 25.5 & 1.9 & 27.9 & $2 \cdot 0$ & 32.4 & 4.1 & $<0.001$ \\
\hline Waist circumference (cm) & $84 \cdot 2$ & 9.4 & 91.9 & 8.2 & $98 \cdot .4$ & 7.8 & $110 \cdot 0$ & $10 \cdot 1$ & $<0.001$ \\
\hline Waist:hip ratio & 0.8 & 0.1 & 0.9 & 0.1 & 0.9 & 0.1 & 1.0 & 0.1 & $<0.001$ \\
\hline Systolic blood pressure (mmHg) & $130 \cdot 7$ & $15 \cdot 5$ & 134.4 & $17 \cdot 6$ & 133.6 & $15 \cdot 5$ & 139.6 & $17 \cdot 8$ & $<0.001$ \\
\hline Diastolic blood pressure $(\mathrm{mmHg})$ & 78.8 & 8.6 & $81 \cdot 1$ & 9.5 & $80 \cdot 8$ & $8 \cdot 3$ & 84.7 & $10 \cdot 2$ & $<0.001$ \\
\hline Whole-blood HbA1c (\%) & 5.6 & 0.5 & 5.6 & 0.4 & $5 \cdot 7$ & 0.6 & 5.9 & 0.8 & $<0.001$ \\
\hline C-reactive protein $(\mathrm{nmol} / \mathrm{l}) \dagger$ & $32 \cdot 3$ & 43.8 & 40.0 & $103 \cdot 8$ & 31.4 & 44.7 & 43.8 & $60 \cdot 9$ & 0.376 \\
\hline Plasma HDL-cholesterol (mmol/l) & 1.9 & 0.5 & 1.8 & 0.5 & 1.7 & 0.4 & 1.5 & 0.4 & $<0.001$ \\
\hline Plasma LDL-cholesterol (mmol/l) & 3.4 & 0.9 & 3.4 & 0.8 & 3.4 & 1.0 & 3.5 & 0.8 & 0.820 \\
\hline Plasma TAG (mmol/l) & 1.2 & 0.8 & 1.2 & 0.6 & 1.4 & 0.6 & 1.7 & 1.1 & $<0.001$ \\
\hline Energy intake $(\mathrm{kJ} / \mathrm{d})$ & 9820 & 2609 & 9527 & 2694 & 9412 & 2570 & 8989 & 2201 & 0.009 \\
\hline Physical activity (MET-h/week) & $116 \cdot 5$ & 67.4 & $105 \cdot 7$ & $60 \cdot 8$ & $115 \cdot 7$ & 79.9 & 96.5 & 57.6 & 0.045 \\
\hline Potatoes $(\mathrm{g} / \mathrm{d})$ & 85.5 & 43.6 & $75 \cdot 7$ & $46 \cdot 3$ & 88.7 & 43.8 & $85 \cdot 8$ & 46.0 & 0.401 \\
\hline Vegetables (g/d) & $210 \cdot 3$ & $112 \cdot 7$ & $197 \cdot 1$ & 87.4 & 193.1 & 67.5 & $195 \cdot 9$ & 84.4 & 0.153 \\
\hline Legumes $(\mathrm{g} / \mathrm{d})$ & 3.5 & 3.6 & $2 \cdot 8$ & $3 \cdot 0$ & 3.4 & 3.6 & 2.9 & $2 \cdot 9$ & 0.390 \\
\hline Fruits $(g / d)$ & 256.9 & $175 \cdot 7$ & $227 \cdot 1$ & 134.7 & $222 \cdot 2$ & 126.9 & $208 \cdot 6$ & 130.9 & 0.005 \\
\hline Dairy products (g/d) & $272 \cdot 2$ & 165.5 & 254.4 & 143.5 & 242.9 & $150 \cdot 0$ & $245 \cdot 0$ & 149.5 & 0.099 \\
\hline Cereals $(g / d)$ & $176 \cdot 3$ & $100 \cdot 4$ & $165 \cdot 8$ & $101 \cdot 6$ & $161 \cdot 0$ & 79.3 & $145 \cdot 0$ & 63.2 & 0.002 \\
\hline Meat $(\mathrm{g} / \mathrm{d})$ & 111.9 & 79.4 & $115 \cdot 8$ & 58.0 & 121.7 & 77.4 & $126 \cdot 8$ & 64.0 & 0.052 \\
\hline Fish $(\mathrm{g} / \mathrm{d})$ & 32.4 & $22 \cdot 6$ & 31.4 & 25.9 & $26 \cdot 6$ & $20 \cdot 2$ & 29.2 & $22 \cdot 0$ & 0.090 \\
\hline Eggs $(g / d)$ & $14 \cdot 2$ & $10 \cdot 0$ & $18 \cdot 7$ & $13 \cdot 1$ & $17 \cdot 2$ & 10.5 & $18 \cdot 9$ & 13.2 & 0.004 \\
\hline Fat and oils $(\mathrm{g} / \mathrm{d})$ & 39.1 & $22 \cdot 0$ & $38 \cdot 3$ & 21.5 & $35 \cdot 1$ & $15 \cdot 0$ & 32.5 & $12 \cdot 7$ & 0.001 \\
\hline Sugar and confectionery $(\mathrm{g} / \mathrm{d})$ & $53 \cdot 6$ & 24.9 & 54.7 & $29 \cdot 7$ & $48 \cdot 7$ & $24 \cdot 7$ & 44.9 & $22 \cdot 0$ & 0.001 \\
\hline Cake $(g / d)$ & $66 \cdot 6$ & $47 \cdot 2$ & 63.4 & 41.4 & 59.9 & $45 \cdot 1$ & $51 \cdot 7$ & $35 \cdot 1$ & 0.002 \\
\hline Non-alcoholic beverages (g/d) & $1935 \cdot 3$ & $872 \cdot 3$ & 1963.2 & 840.9 & $2205 \cdot 3$ & 972.6 & $2061 \cdot 0$ & 911.9 & 0.064 \\
\hline Alcoholic beverages $(\mathrm{g} / \mathrm{d})$ & 209.8 & 288.2 & 217.5 & $272 \cdot 7$ & 273.6 & 453.7 & $240 \cdot 2$ & 384.5 & 0.265 \\
\hline
\end{tabular}

$\mathrm{HbA1c}$, glycated $\mathrm{Hb}$; MET, metabolic equivalent task.

* $P<0.05$ was considered statistically significant. $P$ values are reported two-sided. The median value in each quartile category was used as a variable in a linear regression as a test for trend. $\dagger$ Information available on 358 subjects. 
Table 3. Association between food groups and visceral adipose tissue (VAT) or subcutaneous abdominal adipose tissue (SAAT) in Northern German adults unstratified ${ }^{\star}$ and stratified by BMI (Number of subjects and $\beta$ coefficients)

\begin{tabular}{|c|c|c|c|c|c|c|c|c|c|c|c|c|c|}
\hline \multirow[b]{3}{*}{ Food groups } & \multirow[b]{3}{*}{$n$} & \multicolumn{6}{|c|}{ VAT } & \multicolumn{6}{|c|}{ SAAT } \\
\hline & & \multicolumn{2}{|c|}{ Model 1} & \multicolumn{2}{|c|}{ Model 2} & \multicolumn{2}{|c|}{ Model 3} & \multicolumn{2}{|c|}{ Model 1} & \multicolumn{2}{|c|}{ Model 2} & \multicolumn{2}{|c|}{ Model 3} \\
\hline & & $\beta$ & $P$ & $\beta$ & $P$ & $\beta$ & $P$ & $\beta$ & $P$ & $\beta$ & $P$ & $\beta$ & $P$ \\
\hline \multicolumn{14}{|l|}{ Potatoes } \\
\hline Total & 585 & 0.119 & 0.004 & 0.085 & 0.043 & 0.005 & 0.862 & 0.142 & 0.002 & 0.117 & 0.014 & 0.042 & 0.158 \\
\hline $\mathrm{BMl}<26.7 \mathrm{~kg} / \mathrm{m}^{2}$ & 293 & 0.082 & 0.145 & 0.063 & 0.290 & $<0.001$ & 0.999 & 0.091 & 0.171 & 0.111 & 0.119 & 0.060 & 0.253 \\
\hline $\mathrm{BMI} \geq 26.7 \mathrm{~kg} / \mathrm{m}^{2}$ & 292 & 0.084 & 0.128 & 0.076 & 0.185 & 0.018 & 0.698 & 0.110 & 0.047 & 0.100 & 0.082 & 0.056 & 0.233 \\
\hline $\begin{array}{l}\text { Interaction } \\
\text { Vegetables }\end{array}$ & & & 0.740 & & 0.744 & & 0.269 & & 0.439 & & 0.414 & & 0.182 \\
\hline \multicolumn{14}{|l|}{ Vegetables } \\
\hline $\begin{array}{l}\text { Total } \\
\text { BMl }<26.7 \mathrm{~kg} / \mathrm{m}^{2}\end{array}$ & 585 & 0.008 & 0.824 & 0.004 & 0.914 & 0.020 & 0.433 & -0.012 & 0.770 & -0.023 & 0.620 & -0.027 & 0.371 \\
\hline & 293 & -0.014 & 0.761 & -0.029 & 0.290 & -0.003 & 0.951 & -0.025 & 0.662 & -0.047 & 0.488 & -0.023 & 0.640 \\
\hline $\mathrm{BMI} \geq 26.7 \mathrm{~kg} / \mathrm{m}^{2}$ & 292 & 0.052 & 0.289 & 0.076 & 0.185 & 0.082 & 0.078 & $<0.001$ & 0.992 & 0.005 & 0.929 & -0.044 & 0.346 \\
\hline Interaction & & & 0.191 & & 0.164 & & 0.149 & & 0.600 & & 0.660 & & 0.558 \\
\hline \multicolumn{14}{|l|}{ Legumes } \\
\hline Total & 585 & 0.031 & 0.402 & 0.026 & 0.483 & 0.041 & 0.081 & $<0.001$ & 0.994 & -0.022 & 0.608 & -0.044 & 0.094 \\
\hline $\mathrm{BMl}<26.7 \mathrm{~kg} / \mathrm{m}^{2}$ & 293 & $<0.001$ & 0.992 & 0.015 & 0.766 & 0.015 & 0.696 & -0.015 & 0.809 & $<0.001$ & 0.988 & -0.011 & 0.799 \\
\hline $\mathrm{BMI} \geq 26.7 \mathrm{~kg} / \mathrm{m}^{2}$ & 292 & 0.082 & 0.102 & 0.095 & 0.072 & 0.103 & 0.017 & $<0.001$ & 0.999 & -0.015 & 0.782 & -0.069 & 0.110 \\
\hline Interaction & & & 0.372 & & $<0.001$ & & $<0.001$ & & 0.473 & & $<0.001$ & & 0.001 \\
\hline \multicolumn{14}{|l|}{ Fruits } \\
\hline Total & 585 & -0.067 & 0.069 & -0.082 & 0.053 & -0.038 & 0.159 & -0.060 & 0.148 & -0.065 & 0.178 & 0.008 & 0.803 \\
\hline $\mathrm{BMl}<26.7 \mathrm{~kg} / \mathrm{m}^{2}$ & 293 & -0.064 & 0.196 & -0.107 & 0.073 & -0.083 & 0.060 & -0.024 & 0.679 & -0.042 & 0.550 & 0.044 & 0.402 \\
\hline $\mathrm{BMI} \geq 26.7 \mathrm{~kg} / \mathrm{m}^{2}$ & 292 & -0.024 & 0.635 & -0.057 & 0.328 & -0.018 & 0.704 & -0.037 & 0.467 & -0.067 & 0.248 & -0.034 & 0.471 \\
\hline Interaction & & & 0.173 & & $<0.001$ & & $<0.001$ & & 0.499 & & $<0.001$ & & $<0.001$ \\
\hline \multicolumn{14}{|l|}{ Dairy products } \\
\hline Total & 585 & -0.072 & 0.045 & -0.058 & 0.132 & -0.047 & 0.053 & -0.038 & 0.350 & -0.016 & 0.716 & 0.035 & 0.202 \\
\hline $\mathrm{BMl}<26.7 \mathrm{~kg} / \mathrm{m}^{2}$ & 293 & -0.139 & 0.004 & -0.153 & 0.003 & -0.113 & 0.003 & -0.071 & 0.216 & -0.070 & 0.254 & 0.054 & 0.239 \\
\hline $\mathrm{BMI} \geq 26.7 \mathrm{~kg} / \mathrm{m}^{2}$ & 292 & -0.018 & 0.710 & -0.034 & 0.539 & -0.015 & 0.740 & -0.019 & 0.704 & -0.033 & 0.552 & -0.013 & 0.770 \\
\hline \multirow{2}{*}{\multicolumn{14}{|c|}{ Cereals }} \\
\hline & & & & & & & & & & & & & \\
\hline Total & 585 & -0.088 & 0.041 & -0.132 & 0.045 & -0.062 & 0.136 & -0.093 & 0.054 & $-0 \cdot 101$ & 0.171 & 0.014 & 0.766 \\
\hline $\mathrm{BMl}<26.7 \mathrm{~kg} / \mathrm{m}^{2}$ & 293 & -0.035 & 0.549 & -0.277 & 0.003 & -0.157 & 0.022 & -0.035 & 0.605 & -0.210 & 0.057 & 0.014 & 0.869 \\
\hline $\mathrm{BMI} \geq 26.7 \mathrm{~kg} / \mathrm{m}^{2}$ & 292 & -0.056 & 0.348 & 0.039 & 0.656 & 0.052 & 0.472 & -0.055 & 0.359 & -0.022 & 0.805 & -0.044 & 0.538 \\
\hline Interaction & & & 0.725 & & 0.817 & & 0.704 & & 0.985 & & 0.924 & & 0.752 \\
\hline Meat & & & & & & & & & & & & & \\
\hline Total & 585 & 0.159 & $<0.001$ & 0.084 & 0.083 & 0.011 & 0.714 & 0.159 & 0.001 & 0.106 & 0.053 & 0.032 & 0.353 \\
\hline $\mathrm{BMl}<26.7 \mathrm{~kg} / \mathrm{m}^{2}$ & 293 & 0.008 & 0.894 & -0.110 & 0.091 & -0.009 & 0.850 & -0.109 & 0.123 & -0.177 & 0.023 & -0.088 & 0.124 \\
\hline $\mathrm{BMI} \geq 267 \mathrm{~kg} / \mathrm{m}^{2}$ & 292 & 0.039 & 0.532 & -0.046 & 0.526 & -0.045 & 0.449 & 0.087 & 0.165 & -0.002 & 0.978 & 0.025 & 0.678 \\
\hline Interaction & & & 0.987 & & 0.883 & & 0.306 & & 0.436 & & 0.318 & & 0.155 \\
\hline Red and processed meat & & & & & & & & & & & & & \\
\hline Total & 585 & 0.153 & 0.001 & & & & & 0.148 & 0.004 & & & & \\
\hline $\mathrm{BMl}<26.7 \mathrm{~kg} / \mathrm{m}^{2}$ & 293 & 0.011 & 0.860 & & & & & -0.114 & 0.111 & & & & \\
\hline $\mathrm{BMI} \geq 26 \cdot 7 \mathrm{~kg} / \mathrm{m}^{2}$ & 292 & 0.035 & 0.575 & & & & & 0.085 & 0.177 & & & & \\
\hline Interaction & & & 0.889 & & & & & & 0.545 & & & & \\
\hline White meat & & & & & & & & & & & & & \\
\hline Total & & 0.082 & 0.026 & & & & & 0.100 & 0.017 & & & & \\
\hline $\mathrm{BMl}<26.7 \mathrm{~kg} / \mathrm{m}^{2}$ & 293 & $<0.001$ & 0.997 & & & & & -0.019 & 0.744 & & & & \\
\hline $\mathrm{BMI} \geq 26 \cdot 7 \mathrm{~kg} / \mathrm{m}^{2}$ & 292 & 0.023 & 0.659 & & & & & 0.024 & 0.649 & & & & \\
\hline Interaction & & & 0.680 & & & & & & 0.236 & & & & \\
\hline Fish & & & & & & & & & & & & & \\
\hline & & -0.022 & 0.542 & -0.039 & 0.285 & -0.158 & 0.496 & -0.017 & 0.688 & -0.034 & 0.411 & $<0.001$ & 0.990 \\
\hline $\mathrm{BMl}<26.7 \mathrm{~kg} / \mathrm{m}^{2}$ & 293 & -0.032 & 0.526 & -0.046 & 0.365 & -0.038 & 0.305 & 0.012 & 0.837 & -0.013 & 0.825 & 0.024 & 0.593 \\
\hline $\mathrm{BMI} \geq 26.7 \mathrm{~kg} / \mathrm{m}^{2}$ & 292 & 0.025 & 0.615 & 0.014 & 0.784 & 0.018 & 0.672 & -0.018 & 0.712 & -0.007 & 0.901 & -0.015 & 0.730 \\
\hline Interaction & & & 0.401 & & 0.500 & & 0.134 & & 0.487 & & 0.456 & & 0.126 \\
\hline
\end{tabular}


N British Journal of Nutrition

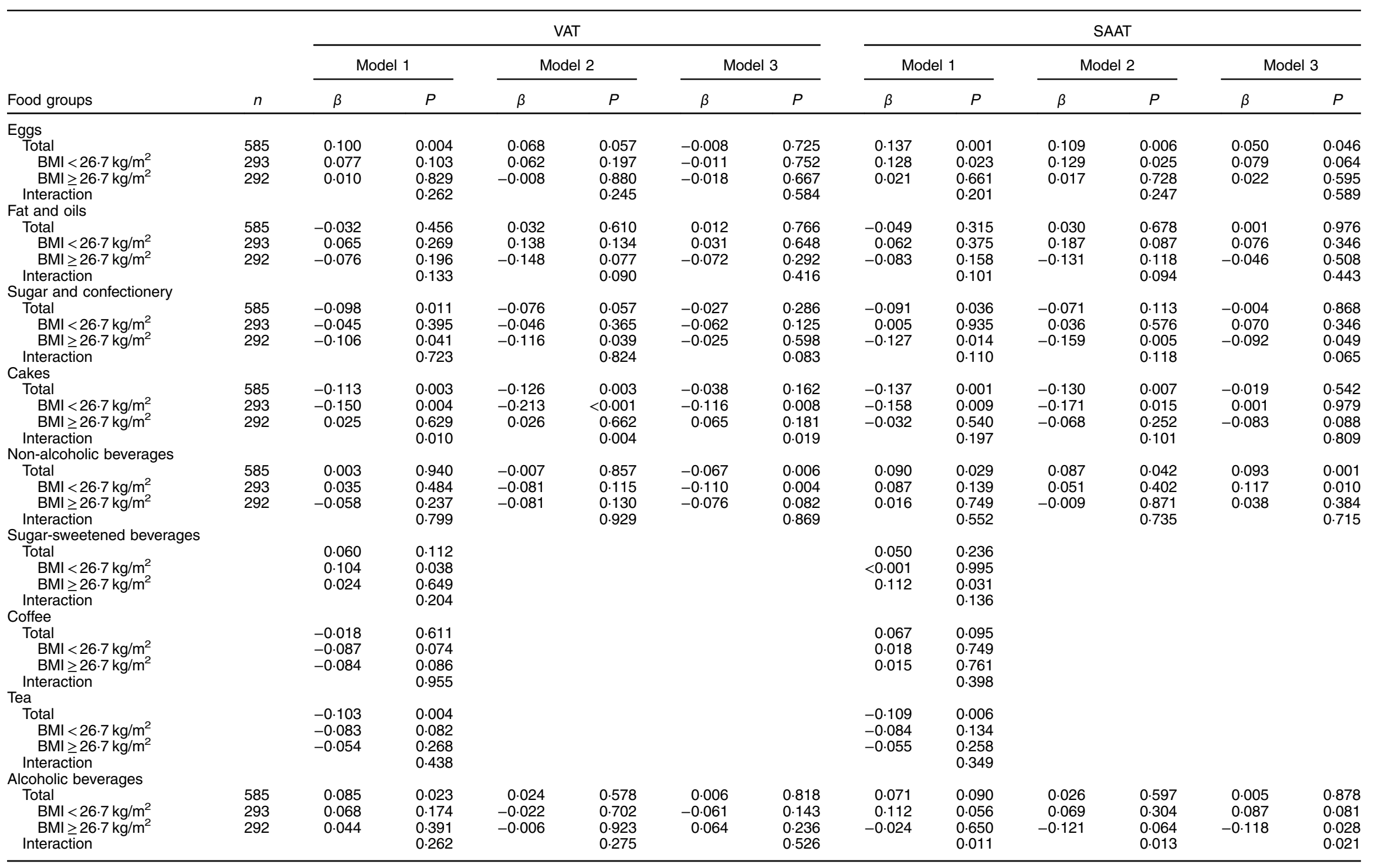

* Model 1 was adjusted for sex, age, physical activity and total energy intake. Model 2 was additionally adjusted for other food groups. Model 3 was model 2 additionally mutually adjusted for VAT or SAAT. $P<0.05$ was considered statistically significant. $P$ values are reported two-sided. 
confectionery, tea and cakes were negatively associated with SAAT. The regression models adjusted for all other food groups confirmed the positive association of intake of potatoes, eggs and non-alcoholic beverages and the inverse association of intake of cakes with SAAT. After further consideration of VAT (model 3), the positive association between eggs and non-alcoholic beverages, respectively, and SAAT remained significant. Stratified by median BMI, the intake of eggs was positively and the intake of cakes inversely associated with SAAT for participants with $\mathrm{BMI}<26.7 \mathrm{~kg} / \mathrm{m}^{2}$, and the intake of potatoes and sugar-sweetened beverages was positively associated with SAAT. Significant effect modification by median BMI was determined for legumes, dairy products and alcoholic beverages.

For the VAT:SAAT ratio, there was a statistically significant inverse association with intakes of non-alcoholic beverages in both models. Consideration of subgroups of non-alcoholic beverage intake revealed a significant inverse association of coffee with the VAT:SAAT ratio in unstratified analysis as well as in the lower BMI stratum. Effect modification by median BMI was observed for intake of sugar-sweetened beverages and the VAT:SAAT ratio (Table 4).

\section{Discussion}

Our data suggest that intakes of potatoes were positively and intakes of cakes negatively associated with total volumes of both VAT and SAAT, after adjusting for potential confounders and considering other food groups. By contrast, intakes of cereals seem to be specifically negatively related to total volumes of VAT, whereas intakes of non-alcoholic beverages and intakes of eggs seem to be specifically positively related to total volumes of SAAT. After mutual adjustment for VAT and SAAT, respectively, the association between eggs and nonalcoholic beverages and SAAT remained significant. Moreover, intakes of non-alcoholic beverages were negatively associated with the VAT:SAAT ratio.

In our study, intakes of potatoes were positively associated with VAT and SAAT but not with the VAT:SAAT ratio. Likewise, in a cohort of adult men and women, the intake of French-fried potatoes was positively associated with waist circumference ${ }^{(11)}$, whereas in another study intake of potatoes was negatively associated with gain in waist circumference in $\operatorname{men}^{(28)}$. These data suggest that the accumulation of AT might be affected by the preparation of food.

Both sweets and cakes were inversely associated with VAT and SAAT in the present study, with a stronger inverse association for intake of cakes with VAT in participants with a BMI $<26.7 \mathrm{~kg} / \mathrm{m}^{2}$. Similarly, an observational study in overweight youths showed a negative association for intakes of sweets with the amount of SAAT but not with VAT $^{(29)}$. Data from the EPIC-Potsdam study suggest a potential benefit of cake and cookie intake on $\mathrm{VAT}^{(30)}$. Inverse associations between intakes of sweets and cakes and AT, however, should be interpreted with regard to possible reporting bias or reverse causation, even if a potential biological mechanism cannot be excluded ${ }^{(30)}$.
Intake of a food group classified as cereals was negatively associated with the volume of VAT in the current study. In a Swedish cross-sectional study, reported intake of pasta was associated with less abdominal fat measured by waist circumference and therefore not further distinguished between VAT and SAAT ${ }^{(11)}$. In a study discriminating between VAT and SAAT, only an inverse association of whole-grain intake with volumes of VAT and SAAT in adults has been reported, whereas the intake of refined grains was positively associated with the volume of $\mathrm{VAT}^{(17)}$.

The intake of non-alcoholic beverages was positively associated with SAAT in the current study, and, after adjustment for SAAT, an inverse association between nonalcoholic beverages and VAT was revealed. Although the inverse association of coffee with VAT or the positive association of coffee with SAAT was not significant, the significant inverse association between coffee and the VAT:SAAT ratio illustrates the tendency to store energy associated with coffee consumption preferably in SAAT adipocytes. VAT has been positively associated with markers of inflammation such as CRP and with decreased levels of adiponectin and elevated levels of leptin ${ }^{(9)}$. In a cross-sectional analysis of Japanese men and women, coffee consumption was shown to be negatively associated with plasma CRP and leptin levels and positively associated with adiponectin ${ }^{(31)}$. Leptin and adiponectin are important adipokines in the regulation of energy balance ${ }^{(9)}$. Therefore, the physiological link between coffee consumption and adipokines might have led to the favourable energy storage in SAAT adipocytes we observed.

The negative association between intakes of non-alcoholic beverages and the VAT:SAAT ratio observed in our study is in contrast to the results of a cross-sectional analysis of 791 non-Hispanic white men and women ${ }^{(32)}$. In the latter study, the proportion of VAT to SAAT increased with increasing intakes of sugar-sweetened beverages, although no significant association with VAT or SAAT was reported ${ }^{(32)}$.

In our study, intakes of meat - both red and processed meat and white meat - were positively associated with VAT and SAAT when the intakes of other food groups were not considered. The associations were stronger for red and processed meat than for white meat. In a study on Latino youth, increase in meat intake (servings/d) were not linked to VAT accumulation $^{(15)}$. Contrary to this, a current meta-analysis of nine prospective studies indicated a positive association between intake of red meat and processed meat and all-cause mortality ${ }^{(33)}$, which might be related to VAT accumulation ${ }^{(34)}$.

In our study, intakes of dairy products were inversely associated with volumes of VAT but not SAAT. However, this association was modified by BMI median with a significant negative association in participants with $\mathrm{BMI}<26.7 \mathrm{~kg} / \mathrm{m}^{2}$ only. Findings on potential benefits of dairy products on the volume of VAT were supported by an observational study showing that dietary Ca intake was inversely associated with changes in VAT over an 1-year period ${ }^{(35)}$. An intervention study investigating the effect of $\mathrm{Ca}$ and vitamin D-supplemented orange juice on weight loss and VAT also showed a significant reduction of VAT in the intervention group compared with the control group, although there was no difference in weight loss between both 
Table 4. Association between food groups and the visceral adipose tissue (VAT):subcutaneous abdominal adipose tissue (SAAT) ratio in Northern German adults unstratified* and stratified by BMI

(Number of subjects and $\beta$ coefficients)

\begin{tabular}{|c|c|c|c|c|c|}
\hline \multirow[b]{3}{*}{ Food groups } & \multirow[b]{3}{*}{$n$} & \multicolumn{4}{|c|}{ VAT:SAAT ratio } \\
\hline & & \multicolumn{2}{|c|}{ Model 1} & \multicolumn{2}{|c|}{ Model 2} \\
\hline & & $\beta$ & $P$ & $\beta$ & $P$ \\
\hline \multicolumn{6}{|l|}{ Potatoes } \\
\hline Total & 585 & -0.006 & 0.834 & 0.003 & 0.940 \\
\hline $\mathrm{BMI}<26.7 \mathrm{~kg} / \mathrm{m}^{2}$ & 293 & 0.041 & 0.389 & 0.004 & 0.941 \\
\hline $\mathrm{BMI} \geq 26.7 \mathrm{~kg} / \mathrm{m}^{2}$ & 292 & -0.005 & 0.916 & -0.004 & 0.931 \\
\hline Interaction & & & 0.676 & & 0.640 \\
\hline \multicolumn{6}{|l|}{ Vegetables } \\
\hline Total & 585 & 0.020 & 0.429 & 0.015 & 0.650 \\
\hline $\mathrm{BMI}<26.7 \mathrm{~kg} / \mathrm{m}^{2}$ & 293 & -0.007 & 0.868 & -0.009 & 0.856 \\
\hline $\mathrm{BMI} \geq 26.7 \mathrm{~kg} / \mathrm{m}^{2}$ & 292 & 0.036 & 0.356 & 0.057 & 0.216 \\
\hline Interaction & & & 0.425 & & 0.343 \\
\hline \multicolumn{6}{|l|}{ Legumes } \\
\hline Total & 585 & 0.034 & 0.191 & 0.052 & 0.089 \\
\hline $\mathrm{BMI}<26.7 \mathrm{~kg} / \mathrm{m}^{2}$ & 293 & 0.009 & 0.844 & 0.010 & 0.815 \\
\hline $\mathrm{BMI} \geq 26.7 \mathrm{~kg} / \mathrm{m}^{2}$ & 292 & 0.069 & 0.086 & 0.090 & 0.033 \\
\hline Interaction & & & 0.933 & & 0.684 \\
\hline \multicolumn{6}{|l|}{ Fruits } \\
\hline Total & 585 & -0.015 & 0.552 & -0.026 & 0.447 \\
\hline $\mathrm{BMI}<26.7 \mathrm{~kg} / \mathrm{m}^{2}$ & 293 & -0.048 & 0.258 & -0.076 & 0.140 \\
\hline $\mathrm{BMI} \geq 26.7 \mathrm{~kg} / \mathrm{m}^{2}$ & 292 & 0.014 & 0.738 & 0.015 & 0.749 \\
\hline Interaction & & & 0.302 & & 0.749 \\
\hline \multicolumn{6}{|l|}{ Dairy products } \\
\hline Total & 585 & -0.042 & 0.094 & -0.038 & $0 \cdot 223$ \\
\hline $\mathrm{BMI}<26.7 \mathrm{~kg} / \mathrm{m}^{2}$ & 293 & -0.069 & 0.095 & -0.094 & 0.035 \\
\hline $\mathrm{BMI} \geq 26.7 \mathrm{~kg} / \mathrm{m}^{2}$ & 292 & 0.010 & 0.800 & 0.012 & 0.777 \\
\hline Interaction & & & 0.196 & & 0.733 \\
\hline \multicolumn{6}{|l|}{ Cereals } \\
\hline Total & 585 & -0.006 & 0.834 & -0.070 & 0.189 \\
\hline $\mathrm{BMI}<26.7 \mathrm{~kg} / \mathrm{m}^{2}$ & 293 & -0.031 & 0.522 & -0.134 & 0.095 \\
\hline $\mathrm{BMI} \geq 26.7 \mathrm{~kg} / \mathrm{m}^{2}$ & 292 & -0.028 & 0.563 & 0.027 & 0.703 \\
\hline Interaction & & & 0.332 & & 0.407 \\
\hline \multicolumn{6}{|l|}{ Meat } \\
\hline Total & 585 & 0.020 & 0.510 & -0.021 & 0.594 \\
\hline $\mathrm{BMI}<26.7 \mathrm{~kg} / \mathrm{m}^{2}$ & 293 & 0.084 & 0.096 & $<0.001$ & 0.999 \\
\hline $\mathrm{BMI} \geq 26.7 \mathrm{~kg} / \mathrm{m}^{2}$ & 292 & -0.032 & 0.524 & -0.022 & 0.703 \\
\hline Interaction & & & 0.166 & & 0.091 \\
\hline \multicolumn{6}{|c|}{ Red and processed meat } \\
\hline Total & 585 & 0.019 & 0.595 & & \\
\hline $\mathrm{BMI}<26.7 \mathrm{~kg} / \mathrm{m}^{2}$ & 293 & 0.089 & 0.085 & & \\
\hline $\mathrm{BMI} \geq 26.7 \mathrm{~kg} / \mathrm{m}^{2}$ & 292 & -0.032 & 0.529 & & \\
\hline Interaction & & & & & \\
\hline White meat & & & & & \\
\hline Total & 585 & -0.0001 & 0.992 & & \\
\hline $\mathrm{BMI}<26.7 \mathrm{~kg} / \mathrm{m}^{2}$ & 293 & 0.028 & 0.502 & & \\
\hline $\mathrm{BMI} \geq 26.7 \mathrm{~kg} / \mathrm{m}^{2}$ & 292 & -0.005 & 0.907 & & \\
\hline Interaction & & & 0.205 & & \\
\hline Fish & & & & & \\
\hline Total & 585 & -0.008 & 0.743 & -0.024 & 0.419 \\
\hline $\mathrm{BMI}<26.7 \mathrm{~kg} / \mathrm{m}^{2}$ & 293 & -0.053 & 0.211 & -0.052 & 0.237 \\
\hline $\mathrm{BMI} \geq 26.7 \mathrm{~kg} / \mathrm{m}^{2}$ & 292 & 0.0214 & 0.589 & -0.001 & 0.975 \\
\hline Interaction & & & 0.200 & & 0.290 \\
\hline Eggs & & & & & \\
\hline Total & 585 & -0.021 & 0.386 & -0.022 & 0.451 \\
\hline $\mathrm{BMI}<26.7 \mathrm{~kg} / \mathrm{m}^{2}$ & 293 & -0.011 & 0.790 & -0.028 & 0.503 \\
\hline $\mathrm{BMI} \geq 26.7 \mathrm{~kg} / \mathrm{m}^{2}$ & 292 & -0.001 & 0.973 & -0.015 & 0.717 \\
\hline Interaction & & & 0.847 & & 0.723 \\
\hline Fat and oils & & & & & \\
\hline Total & 585 & -0.004 & 0.906 & 0.011 & 0.830 \\
\hline $\mathrm{BMI}<26.7 \mathrm{~kg} / \mathrm{m}^{2}$ & 293 & -0.115 & 0.818 & -0.035 & 0.661 \\
\hline $\mathrm{BMI} \geq 26.7 \mathrm{~kg} / \mathrm{m}^{2}$ & 292 & -0.010 & 0.837 & -0.002 & 0.981 \\
\hline Interaction & & & 0.652 & & 0.565 \\
\hline Sugar and confectione & & & & & \\
\hline Total & 585 & -0.019 & 0.465 & -0.003 & 0.922 \\
\hline $\mathrm{BMI}<26.7 \mathrm{~kg} / \mathrm{m}^{2}$ & 293 & -0.048 & 0.290 & -0.079 & 0.096 \\
\hline
\end{tabular}


Table 4 Continued

\begin{tabular}{|c|c|c|c|c|c|}
\hline \multirow[b]{3}{*}{ Food groups } & \multirow[b]{3}{*}{$n$} & \multicolumn{4}{|c|}{ VAT:SAAT ratio } \\
\hline & & \multicolumn{2}{|c|}{ Model 1} & \multicolumn{2}{|c|}{ Model 2} \\
\hline & & $\beta$ & $P$ & $\beta$ & $P$ \\
\hline $\begin{array}{c}\mathrm{BMI} \geq 26.7 \mathrm{~kg} / \mathrm{m}^{2} \\
\text { Interaction }\end{array}$ & 292 & 0.027 & $\begin{array}{l}0.514 \\
0.265\end{array}$ & 0.054 & $\begin{array}{l}0.235 \\
0.205\end{array}$ \\
\hline \multicolumn{6}{|l|}{ Cakes } \\
\hline Total & 585 & 0.009 & 0.748 & -0.025 & 0.462 \\
\hline $\mathrm{BMl}<26.7 \mathrm{~kg} / \mathrm{m}^{2}$ & 293 & -0.029 & 0.510 & -0.103 & 0.043 \\
\hline $\mathrm{BMI} \geq 26.7 \mathrm{~kg} / \mathrm{m}^{2}$ & 292 & 0.036 & 0.388 & 0.067 & 0.162 \\
\hline Interaction & & & 0.297 & & 0.267 \\
\hline \multicolumn{6}{|c|}{ Non-alcoholic beverages } \\
\hline Total & 585 & -0.081 & 0.001 & -0.010 & 0.001 \\
\hline $\mathrm{BMI}<26.7 \mathrm{~kg} / \mathrm{m}^{2}$ & 293 & -0.113 & 0.007 & -0.141 & 0.002 \\
\hline $\mathrm{BMI} \geq 26.7 \mathrm{~kg} / \mathrm{m}^{2}$ & 292 & -0.063 & 0.109 & -0.060 & 0.162 \\
\hline Interaction & & & 0.828 & & 0.890 \\
\hline \multicolumn{6}{|c|}{ Sugar-sweetened beverages } \\
\hline Total & & 0.018 & 0.554 & & \\
\hline $\mathrm{BMl}<26.7 \mathrm{~kg} / \mathrm{m}^{2}$ & & 0.111 & 0.009 & & \\
\hline $\mathrm{BMI} \geq 26.7 \mathrm{~kg} / \mathrm{m}^{2}$ & & -0.068 & 0.103 & & \\
\hline Interaction & & & 0.002 & & \\
\hline \multicolumn{6}{|l|}{ Coffee } \\
\hline Total & & $-0 \cdot 108$ & $<0.001$ & & \\
\hline $\mathrm{BMI}<26.7 \mathrm{~kg} / \mathrm{m}^{2}$ & & -0.131 & 0.001 & & \\
\hline $\mathrm{BMI} \geq 26 \cdot 7 \mathrm{~kg} / \mathrm{m}^{2}$ & & -0.075 & 0.054 & & \\
\hline Interaction & & & 0.346 & & \\
\hline \multicolumn{6}{|l|}{ Tea } \\
\hline Total & & -0.007 & 0.791 & & \\
\hline $\mathrm{BMl}<26.7 \mathrm{~kg} / \mathrm{m}^{2}$ & & -0.019 & 0.633 & & \\
\hline $\mathrm{BMI} \geq 26 \cdot 7 \mathrm{~kg} / \mathrm{m}^{2}$ & & $<0.001$ & 0.983 & & \\
\hline Interaction & & & 0.942 & & \\
\hline \multicolumn{6}{|l|}{ Alcoholic beverages } \\
\hline Total & 585 & 0.024 & 0.359 & 0.009 & 0.809 \\
\hline $\mathrm{BMl}<26.7 \mathrm{~kg} / \mathrm{m}^{2}$ & 293 & $\begin{array}{r}0.024 \\
-0.023\end{array}$ & 0.586 & -0.092 & 0.061 \\
\hline $\mathrm{BMI} \geq 26.7 \mathrm{~kg} / \mathrm{m}^{2}$ & 292 & $\begin{array}{r}-0.076 \\
0.076\end{array}$ & 0.065 & $\begin{array}{r}-0.052 \\
0.119\end{array}$ & 0.023 \\
\hline Interaction & & & 0.191 & & 0.211 \\
\hline
\end{tabular}

* Model 1 was adjusted for sex, age, physical activity and total energy intake. Model 2 was additionally adjusted for other food groups. $P<0.05$ was considered statistically significant. $P$ values are reported two-sided.

groups $^{(36)}$. Moreover, the intake of low-fat dairy products was negatively associated with waist circumference in a Swedish cohort $^{(11)}$. In a randomised controlled trial on obese Japanese, VAT and SAAT were reduced in a group drinking dissolved milk protein at breakfast compared with a group with intake of soya protein $^{(37)}$. In our study, however, the inverse association between intakes of dairy products and VAT remained no longer significant after considering the intake of other food groups. This observation might be explained because high intake of dairy products may reflect a dietary pattern being associated with VAT, and a separation of effects on VAT accumulation in multivariable regression by adjusting for intake of other food groups might thus miss associations between dairy products and AT volume.

In line with our findings, in two other cross-sectional studies, alcohol consumption was positively associated with $\operatorname{VAT}^{(38,39)}$. Our study, however, does not support the observed inverse association between alcohol intake and SAAT reported in one of the two studies on women ${ }^{(39)}$.

We observed a negative trend of reported energy intakes with quartiles of VAT and SAAT. Besides, the mean value of BMI in our study sample was $>25 \mathrm{~kg} / \mathrm{m}^{2}$, and 125 study participants had a BMI $\geq 30 \mathrm{~kg} / \mathrm{m}^{2}$. Similarly, in the EPIC-Potsdam study, BMI and related measures of obesity, such as body fat and body weight, were major determinants of under-reporting in studies using self-administered $\mathrm{FFQ}^{(40)}$, indicating that the negative association we observed between VAT or SAAT and reported energy intakes was possibly because of underreporting of food intake in our study population.

According to a current systematic review summarising the evidence for qualitative aspects of diet and VAT and SAAT, about half of all observational studies did control their analysis for BMI or measures of regional adiposity, whereas the other half did not ${ }^{(18)}$. Although there might be cases where a high BMI is caused by high lean muscle mass and is not related to a high volume of total AT, in our study we observed a strong correlation between both total volumes of SAAT and VAT and BMI. In observational studies, waist circumference is commonly used as an anthropometric surrogate for VAT. Correspondingly, waist circumference and VAT were highly correlated $(r$ 0.84; $P<0.0001)$ in our study. Therefore, adjustment for BMI or measures of regional adiposity was probably not appropriate in our study. Additionally, because of high correlation between AT, mutual adjustment for VAT and SAAT, respectively, has been debated. Only in half of the studies investigating the effect of diet on AT, both VAT and SAAT had been assessed actually 
allowing mutual adjustment ${ }^{(18)}$. Even in the same cohort, one study used mutual AT adjustment ${ }^{(17)}$, whereas the other did not ${ }^{(39)}$.

To our knowledge, the present study is the first observational study investigating the association between overall food group intake and total volumes of directly measured VAT and SAAT, and, next to studies on the Framingham cohort, the current study is the only population-based study relating diet to AT determined by whole-body volumetric scans ${ }^{(17,39)}$. CT and MRI are considered the gold standards for direct and differentiated assessment of AT compartments and are more favourable than the other less specific and accurate imaging techniques such as dual-energy X-ray absorptiometry or ultrasound. Most population-based studies investigating the effect of diet on VAT or SAAT, however, only used single-slice or few- to multipleslice abdominal MRI or CT scans instead of scans covering the whole abdomen. The use of single-slice images for the estimation of AT volumes, however, is less accurate, especially because data of the slice area representing the highest correlation with abdominal AT might be affected by intrasubject variability ${ }^{(41,42)}$. In the Framingham cohort, less total volume of VAT compared with the volumes of VAT in our study population has been reported ${ }^{(17,39)}$. This might be related to the lower mean age in the Framingham cohort compared with the PopGen control cohort and the positive correlation between age and VAT in our study $(r 0 \cdot 18 ; P<0 \cdot 0001)$.

Major strengths of our study include the assessment of total volumes of VAT and SAAT by MRI covering the whole abdomen in a large population-based sample, the use of an unbiased MRI post-processing approach including the validation of the AT assessment using the 'gold standard' of manual determination of VAT and SAAT, and the consideration of important covariates such as physical activity.

Still, our study has several limitations. Because of the cross-sectional design, the associations we observed may not be causal and may suffer from reverse causation. Furthermore, the current FFQ has not been validated so far. The FFQ used in the PopGen control cohort is based on the FFQ developed for the EPIC-Potsdam study. The macronutrient and energy intakes of the EPIC-Potsdam FFQ have been validated against data from 24-h dietary recalls, urinary excretion and doubly labelled water measurements, which showed an acceptable relative validity ${ }^{(23)}$. Adapted from the EPIC-Potsdam FFQ, the PopGen FFQ comprises the food items that have been shown to discriminate the most between study participants with regard to absolute intake of food items and nutrients, suggesting an acceptable relative validity as well. Nevertheless, the FFQ might be affected by measurement error and limited accuracy in assessment of food intake. Moreover, because our study participants had to fill out a self-administered FFQ to assess food intake, we could not rule out that the observed associations were biased by dietary misreporting. Similarly, we could not exclude the possibility of response bias or selection bias due to the MRI exclusion criteria. Further, dairy products, alcoholic beverages and non-alcoholic beverages are not associated with VAT and SAAT in the same way. Therefore, to take into account possible interactions between foods, studies investigating the association between AT and dietary patterns are warranted.
Finally, although the PopGen control cohort is based on a random sample of the general population, generalisability of our results may be debatable because all participants had a similar ethnic and cultural Northern German background.

In conclusion, our results indicate that intake of individual food groups may be independently predictive of the total amount of VAT or SAAT and the proportion of VAT to SAAT, with the observed associations being dependent on BMI. Our research encourages further investigation into qualitative aspects of food intake and abdominal obesity, with consideration of effect modification by BMI.

\section{Acknowledgements}

This study was supported by grants from the Deutsche Forschungsgemeinschaft Excellence Cluster 'Inflammation at Interfaces' (EXC306, EXC306/2) and from the German Federal Ministry of Education and Research (01GR0468). The PopGen 2.0 network is supported by a grant from the German Ministry for Education and Research (01EY1103).

The authors' responsibilities were as follows: D. R., K. F., M. K. and U. N. designed research; M. K., G. J., U. N. and W. L. collected or provided data; D. R. quantified AT variables; H.-P. M. and J. K. provided methodological adaptions of the ATLAS-software; D. R. analysed the data and wrote the manuscript. All authors critically reviewed the manuscript and approved its final version.

The authors declare no potential conflicts of interest.

\section{References}

1. Tchernof A \& Despres JP (2013) Pathophysiology of human visceral obesity: an update. Physiol Rev 93, 359-404.

2. Patel P \& Abate N (2013) Role of subcutaneous adipose tissue in the pathogenesis of insulin resistance. J Obes $\mathbf{2 0 1 3}, 489187$.

3. Neeland IJ, Ayers CR, Rohatgi AK, et al. (2013) Associations of visceral and abdominal subcutaneous adipose tissue with markers of cardiac and metabolic risk in obese adults. Obesity 21, E439-E447.

4. Manolopoulos KN, Karpe F \& Frayn KN (2010) Gluteofemoral body fat as a determinant of metabolic health. Int $J$ Obes (Lond) 34, 949-959.

5. Eastwood SV, Tillin T, Wright A, et al. (2014) Thigh fat and muscle each contribute to excess cardiometabolic risk in South Asians, independent of visceral adipose tissue. Obesity 22, 2071-2079.

6. Golan R, Shelef I, Rudich A, et al. (2012) Abdominal superficial subcutaneous fat: a putative distinct protective fat subdepot in type 2 diabetes. Diabetes Care 35, 640-647.

7. Fox CS, Massaro JM, Hoffmann U, et al. (2007) Abdominal visceral and subcutaneous adipose tissue compartments: association with metabolic risk factors in the Framingham Heart Study. Circulation 116, 39-48.

8. Kaess BM, Pedley A, Massaro JM, et al. (2012) The ratio of visceral to subcutaneous fat, a metric of body fat distribution, is a unique correlate of cardiometabolic risk. Diabetologia $\mathbf{5 5}$, 2622-2630.

9. Ibrahim MM (2010) Subcutaneous and visceral adipose tissue: structural and functional differences. Obes Rev 11, 11-18.

10. Ghosh A, Bose K \& Das Chaudhuri AB (2003) Association of food patterns, central obesity measures and metabolic risk 
factors for coronary heart disease (CHD) in middle aged Bengalee Hindu men, Calcutta, India. Asia Pac J Clin Nutr 12, 166-171.

11. Krachler B, Eliasson M, Stenlund H, et al. (2006) Reported food intake and distribution of body fat: a repeated cross-sectional study. Nutr J 5, 34 .

12. Thomas EL, Parkinson JR, Frost GS, et al. (2012) The missing risk: MRI and MRS phenotyping of abdominal adiposity and ectopic fat. Obesity (Silver Spring) 20, 76-87.

13. Ludescher B, Machann J, Eschweiler GW, et al. (2009) Correlation of fat distribution in whole body MRI with generally used anthropometric data. Invest Radiol 44, 712-719.

14. Ventura E, Davis J, Byrd-Williams C, et al. (2009) Reduction in risk factors for type 2 diabetes mellitus in response to a lowsugar, high-fiber dietary intervention in overweight Latino adolescents. Arch Pediatr Adolesc Med 163, 320-327.

15. Davis JN, Alexander KE, Ventura EE, et al. (2009) Inverse relation between dietary fiber intake and visceral adiposity in overweight Latino youth. Am J Clin Nutr 90, 1160-1166.

16. Parikh S, Pollock NK, Bhagatwala J, et al. (2012) Adolescent fiber consumption is associated with visceral fat and inflammatory markers. J Clin Endocrinol Metab 97, E1451-E1457.

17. McKeown NM, Troy LM, Jacques PF, et al. (2010) Whole- and refined-grain intakes are differentially associated with abdominal visceral and subcutaneous adiposity in healthy adults: the Framingham Heart Study. Am J Clin Nutr 92, 1165-1171.

18. Fischer K, Pick JA, Moewes D, et al. (2015) Qualitative aspects of diet affecting visceral and subcutaneous abdominal adipose tissue: a systematic review of observational and controlled intervention studies. Nutr Rev 73, 191-215.

19. Krawczak M, Nikolaus S, von Eberstein H, et al. (2006) PopGen: population-based recruitment of patients and controls for the analysis of complex genotype-phenotype relationships. Community Genet 9, 55-61.

20. Nothlings U \& Krawczak M (2012) PopGen: a populationbased biobank with prospective follow-up of a control group. Bundesgesundheitsblatt, Gesundheitsforschung, Gesundheitsschutz 55, 831-835.

21. Nothlings U, Hoffmann K, Bergmann MM, et al. (2007) Fitting portion sizes in a self-administered food frequency questionnaire. J Nutr 137, 2781-2786.

22. Dehne LI, Klemm C, Henseler G, et al. (1999) The German Food Code and Nutrient Data Base (BLS II.2). Eur J Epidemiol 15, 355-359.

23. Kroke A, Klipstein-Grobusch K, Voss S, et al. (1999) Validation of a self-administered food-frequency questionnaire administered in the European Prospective Investigation into Cancer and Nutrition (EPIC) study: comparison of energy, protein, and macronutrient intakes estimated with the doubly labeled water, urinary nitrogen, and repeated 24-h dietary recall methods. Am J Clin Nutr 70, 439-447.

24. Fischer K, Moewes D, Koch M, et al. (2015) MRI-determined total volumes of visceral and subcutaneous abdominal and trunk adipose tissue are differentially and sex-dependently associated with patterns of estimated usual nutrient intake in a northern German population. Am J Clin Nutr 101, 794-807.

25. Koch M, Borggrefe J, Barbaresko J, et al. (2014) Dietary patterns associated with magnetic resonance imaging-determined liver fat content in a general population study. Am J Clin Nutr 99, 369-377.
26. Muller HP, Raudies F, Unrath A, et al. (2011) Quantification of human body fat tissue percentage by MRI. NMR Biomed $\mathbf{2 4}$, $17-24$.

27. Lindauer E, Dupuis L, Muller HP, et al. (2013) Adipose tissue distribution predicts survival in amyotrophic lateral sclerosis. PLOS ONE 8, e67783.

28. Halkjaer J, Sorensen TI, Tjonneland A, et al. (2004) Food and drinking patterns as predictors of 6-year BMI-adjusted changes in waist circumference. Br J Nutr 92, 735-748.

29. Hairston KG, Vitolins MZ, Norris JM, et al. (2012) Lifestyle factors and 5-year abdominal fat accumulation in a minority cohort: the IRAS Family Study. Obesity (Sliver Spring) 20, 421-427.

30. von Ruesten A, Feller S, Bergmann MM, et al. (2013) Diet and risk of chronic diseases: results from the first 8 years of follow-up in the EPIC-Potsdam study. Eur J Clin Nutr 67, 412-419.

31. Yamashita K, Yatsuya H, Muramatsu T, et al. (2012) Association of coffee consumption with serum adiponectin, leptin, inflammation and metabolic markers in Japanese workers: a cross-sectional study. Nutr Diabetes 2, e33.

32. Odegaard AO, Choh AC, Czerwinski SA, et al. (2012) Sugarsweetened and diet beverages in relation to visceral adipose tissue. Obesity (Sliver Spring) 20, 689-691.

33. Larsson SC \& Orsini N (2014) Red meat and processed meat consumption and all-cause mortality: a meta-analysis. Am J Epidemiol 179, 282-289.

34. Rosenquist KJ, Massaro JM, Pedley A, et al. (2015) Fat quality and incident cardiovascular disease, all-cause mortality and cancer mortality. J Clin Endocrinol Metab 100, 227-234.

35. Bush NC, Alvarez JA, Choquette SS, et al. (2010) Dietary calcium intake is associated with less gain in intra-abdominal adipose tissue over 1 year. Obesity (Sliver Spring) 18, 2101-2104

36. Rosenblum JL, Castro VM, Moore CE, et al. (2012) Calcium and vitamin D supplementation is associated with decreased abdominal visceral adipose tissue in overweight and obese adults. Am J Clin Nutr 95, 101-108.

37. Takahira M, Noda K, Fukushima M, et al. (2011) Randomized, double-blind, controlled, comparative trial of formula food containing soy protein vs. milk protein in visceral fat obesity FLAVO study. Circ J 75, 2235-2243.

38. Kim KH, Oh SW, Kwon H, et al. (2012) Alcohol consumption and its relation to visceral and subcutaneous adipose tissues in healthy male Koreans. Ann Nutr Metab 60, 52-61.

39. Molenaar EA, Massaro JM, Jacques PF, et al. (2009) Association of lifestyle factors with abdominal subcutaneous and visceral adiposity: the Framingham Heart Study. Diabetes Care 32, 505-510.

40. Voss S, Kroke A, Klipstein-Grobusch K, et al. (1997) Obesity as a major determinant of underreporting in a selfadministered food frequency questionnaire: results from the EPIC-Potsdam Study. Z Ernabrungswiss 36, 229-236.

41. Greenfield JR, Samaras K, Chisholm DJ, et al. (2002) Regional intra-subject variability in abdominal adiposity limits usefulness of computed tomography. Obes Res 10, 260-265.

42. Shen W, Punyanitya M, Wang Z, et al. (2004) Visceral adipose tissue: relations between single-slice areas and total volume. Am J Clin Nutr 80, 271-278. 